\title{
Mistaken Identity of Popliteal Artery Pseudoaneurysm for Deep Vein Thrombosis: a Case Report
}

\author{
T.G. Rashid*, Z. Andreou, M.J. Metcalfe, A.J. Marcus, K. Lotzof and R. le R. Bird \\ Department of Vascular Surgery, Barnet General Hospital, Barnet, London, Herts, UK
}

\begin{abstract}
Popliteal artery aneurysms are often incorrectly diagnosed and subsequently mismanaged with resultant preventable complications. We present a case of a swollen lower extremity clinically resembling a deep vein thrombosis (DVT), following tibial fracture fixation. It was treated as a DVT on the basis of ultrasound imaging at a different hospital. Subsequent imaging showed a popliteal artery pseudoaneurysm for which the patient required 'acute' surgical intervention. DVTs and popliteal artery aneurysms and pseudoaneurysms may coexist. We stress the importance of searching for them in patients with suspected DVT, despite an ultrasound scan (USS) which is negative or positive for thrombosis.
\end{abstract}

Keywords: Popliteal artery aneurysm, pseudoaneurysm, false aneurysm, misdiagnosis, deep vein thrombosis.

\section{BACKGROUND}

Pseudo- or false aneurysms are localized dilatations of an artery where there is a contained rupture through the two innermost layers of the vessel wall. Although popliteal artery aneurysms are the most common peripheral aneurysm, their incidence is low at $0.1-1 \%$ [1]. $50 \%$ are bilateral [2]. The incidence of pseudoaneurysms of the popliteal artery is as yet not reported in the literature.

It is generally recognized that pseudoaneurysms most commonly result from catheterization of an artery (most frequently the common femoral artery during angiographic procedures). Other causes include: a slow anastomotic leak in arterial bypass grafts [3] and dialysis access fistulae, arterial wall infections, following an overaggressive endarterectomy [4], intravenous drug abuse [5], blunt trauma [6], penetrating injury $[7,8]$ and following traumatic fracture, fracture fixation and joint replacement surgery $[9$, $10]$.

The clinical diagnosis of a popliteal artery aneurysm remains problematic due to difficulty in accurately examining the popliteal fossa and often a low index of suspicion. The commonest acute presentation of a popliteal aneurysm is ischaemia. Popliteal pseudoaneurysms however present with pain, swelling and rupture. Due to their large size they may cause compression of local structures including the popliteal vein and tibial nerve.

Delayed or incorrect diagnosis of a popliteal pseudoaneurysm may result in varying complications: distal oedema of the extremity, acute haemorrhage into the aneurysm, distal emboli, acute ischaemia and nerve palsy

*Address correspondence to this author at the Department of Vascular Surgery, Barnet General Hospital, UK; Tel: 07769703553;

Fax: 0208907 9032; E-mail: dr.tina.rashid@doctors.org.uk
[11]. Aneurysm rupture [12] can exist with or indeed cause acute venous thrombosis of the popliteal vein $[13,14]$ and may require amputation of the affected limb. Literature to date describes the difficulty in diagnosis of true popliteal aneurysms, femoral and popliteal aneurysms presenting in association with venous thromboses and one report of a femoral artery pseudoaneurysm misdiagnosed as a DVT [15]. There are two reported cases of patients with bilateral popliteal artery aneurysms misdiagnosed and treated as a DVT, despite negative venography [13].

We report a case of a popliteal artery pseudoaneurysm initially diagnosed and treated as a DVT which required subsequent surgical intervention.

\section{CASE PRESENTATION}

A 68-year old gentleman involved in a road traffic accident in India sustained a grade I compound fracture of the proximal one third of the right tibia and fibula. He was managed with an interlocking nail with three proximal and one distal locking screws and a transverse plate for the tibial tubercle fragment. Three months post-surgery, he presented to a UK hospital with a one week history of an oedematous, swollen painful leg. An ultrasound scan (USS) diagnosed a 'below-knee' DVT for which he was warfarinised. One month later, he presented to our hospital with worsening leg swelling despite adequate anticoagulation. A repeat USS revealed a pulsating mass of approximately $10 \mathrm{~cm}$ in the popliteal fossa and no DVT was seen. A CT angiogram suggested the presence of a pseudoaneurysm (Fig. 1) but was inconclusive due to image interference from the metalwork within the tibia. The diagnosis of a $7 \mathrm{~cm}$ diameter pseudoaneurysm was subsequently confirmed with a transfemoral angiogram (Fig. 2). His haemoglobin dropped from $9.6 \mathrm{~g} / \mathrm{dL}$ on admission to $7.9 \mathrm{~g} / \mathrm{dL}$ within 48 hours of admission with no other source of blood loss and he required a blood transfusion. Blood loss was therefore thought to be a result of a mixture of haemodilution and sequestration of blood within the pseudoaneurysm. He underwent emergency surgical repair of his pseudoaneurysm via a medial approach. 

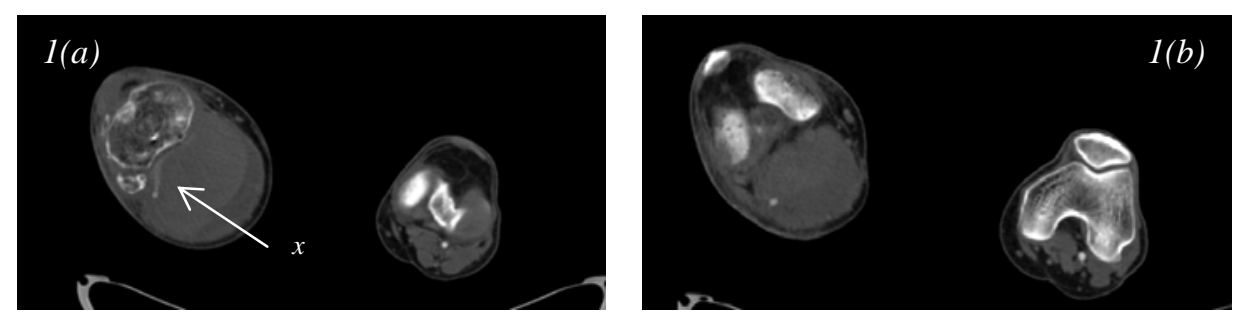

Fig. (1). (a) and (b): (a) contrast leak from popliteal artery into contained wall of pseudoaneurysm.(x); (b) surrounding haematoma within pseudoaneurysm sac.

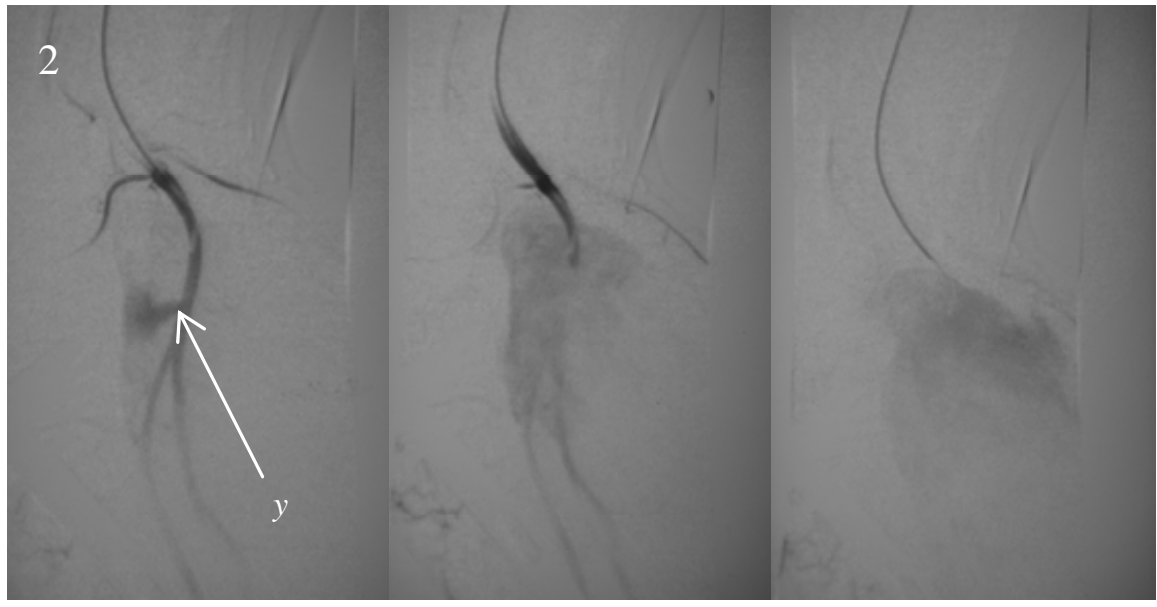

Fig. (2). Angiogram used to confirm leak of contrast from popliteal artery into sac of pseudoaneurysm (y).

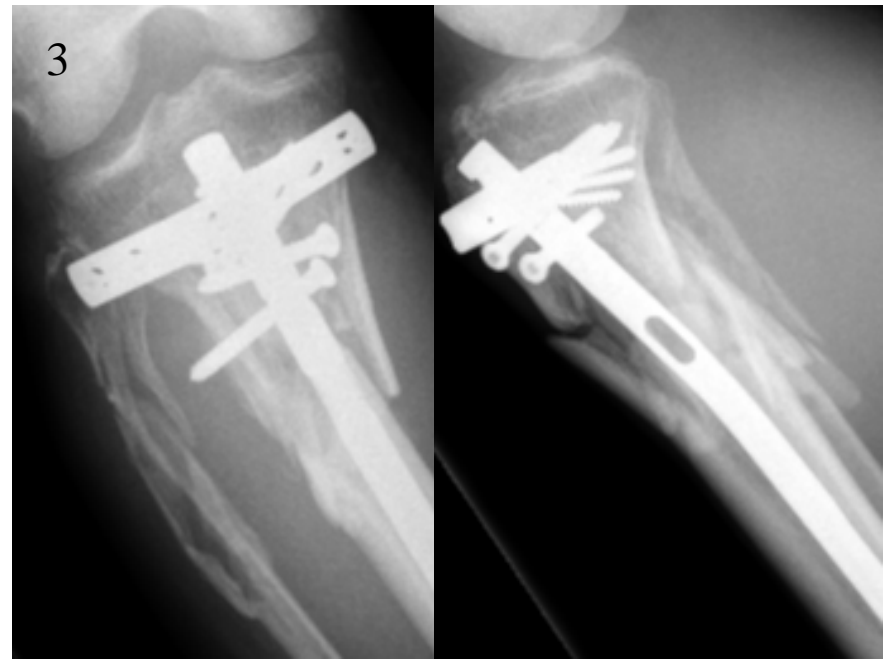

Fig. (3). AP and lateral radiographs of the tibia demonstrating the proximity of both the original fracture site and the interlocking screws to the popliteal artery and pseudoaneurysm.

The pseudoaneurysm was located close to both the tip of a proximal interlocking screw and the original fracture site (Fig. 3). A large haematoma of approximately 500mls was evacuated, the aneurysmal arterial defect closed via direct suturing with 5.0 prolene. He had an uneventful recovery and was discharged 5 days later.

\section{CONCLUSION}

We have reported a case of popliteal artery pseudoaneurysm following orthopaedic surgery which was misdiagnosed as a DVT. Subsequent anticoagulation resulted in progressive haemorrhage into the pseudoaneurysm and required emergency surgical intervention to prevent irreversible complications. We suspect that either the initial DVT diagnosis on USS was incorrect or that the deep vein had recanalised with warfarin therapy.

Early accurate diagnosis of popliteal artery aneurysms is made less frequently than it should be [16]. A pseudoaneurysm can be distinguished on ultrasound from a true aneurysm by visualization of a neck or hole feeding the 
pseudoaneurysm, confirming a defect in part of the vessel wall. Harris and Barrie [13] have described two cases of popliteal artery aneurysms, both diagnosed and treated as DVT despite normal venography. In one case, this gave rise to preventable complications (left calf claudication probably amenable to surgery if diagnosed earlier) and in both cases, led to a delay in the diagnosis. In our case, the diagnosis of DVT was confirmed at a different hospital on USS.

There are data to suggest that popliteal artery aneurysms can be associated with venous disease. Giustra et al analysed 623 cases of popliteal artery aneurysms and found the incidence of venous disease (compression or thrombosis of popliteal veins and venous thrombosis) to be $10-15 \%$ [14], using a combination of ultrasound, xeroradiography, preand intra-operative femoral arteriography. However, the exact incidence was difficult to establish due to the lack of confirmatory venographic studies. They have suggested that preoperative venography be undertaken in all cases of popliteal artery aneurysm.

Accurate clinical examination of the popliteal fossa is difficult [16]. There is evidence to suggest that all symptomatic as well as asymptomatic popliteal aneurysms above $2 \mathrm{~cm}$ in diameter should be surgically treated [17]. A twenty-year experience of 147 popliteal aneurysms has shown that complications occur in $64 \%$ and in $36 \%$ of these, result in amputation [2]. Those patients who present with clinical signs and symptoms but a negative USS for DVT often do not get given a diagnosis. We stress the importance of specifically seeking a popliteal artery aneurysm in an USS either positive or negative for a DVT.

Traumatic fractures and penetrating trauma require a high index of suspicion for pseudoaneurysm formation not only at initial diagnosis, but also during fracture reduction and fixation. In these patients, DVT may be a common cause for leg swelling, but pseudoaneurysm formation must also be considered and actively excluded.

USS is a quick and cheap tool and in the hands of a clinician expert in ultrasound diagnosis may aid in reducing preventable complications resulting from missed diagnosis of popliteal artery aneurysms and pseudoaneurysms.

\section{CONSENT}

Written informed consent was obtained from the patient for publication of this case report and any accompanying images. A copy of the written consent is available for review by the Editor-in-Chief of this journal.

\section{AUTHORS' CONTRIBUTIONS}

TR, ZA and MM drafted the original manuscript. AM and KL were involved in the selection of images used. RleB approved the final manuscript.

\section{ACKNOWLEDGEMENTS}

Many thanks to the Medical Education Departments at Barnet Hospital.

\section{ABBREVIATIONS}

$\begin{array}{lll}\text { DVT } & = & \text { Deep vein thrombosis } \\ \text { USS } & =\text { Ultrasound }\end{array}$

\section{REFERENCES}

[1] Ohrlander, T.; Holst, J.; Malina, M. Emergency intervention for thrombosed popliteal artery aneurysm: can the limb be salvaged? $J$. Cardiovasc. Surg. (Torino), 2007, 48(3), 289-297.

[2] Vermilion, B.D.; Kimmins, S.A.; Pace, W.G.; Evans, W.E. A review of one hundred and forty-seven popliteal aneurysms with long term follow-up. Surgery, 1981, 90, 1009-1014.

[3] Skourtis, G.; Bountouris, I.; Papacharalambous, G.; Mahera, E.; Besias, N.; Antoniou, I.; Pavlidis, P.; Maras, D.; Panoussis, P.; Andrikopoulos, V. Anastomotic pseudoaneurysms: our experience with 49 cases. Ann. Vasc. Surg., 2006, 20(5), 582-589.

[4] Ilijevski, N.S.; Gajin, P.; Neskovic, V.; Kolar, J.; Radak, D. Postendarterectomy common carotid artery pseudoaneurysm. Vascular, 2006, 14(3), 177-180.

[5] Coughlin, P.A.; Mavor, A.I. Arterial consequences of recreational drug use. Eur. J. Vasc. Endovasc. Surg., 2006, 32(4), 389-396.

[6] Davidovia, B.L.; Vasia, M.D.; Markovia, M.D.; Sindjelia, P.R.; Pavlovia, U.S.; Kuzmanovic, B.I. Carotid artery false aneurysm caused by blunt trauma. A case report: Int. Angiol., 2007, 26(1), 72-74.

[7] Kakar, B.K.; Babar, K.M.; Khan, A.; Naeem, M.; Khan, I. Surgical experience of post-traumatic pseudo aneurysm of the brachial artery at a tertiary care hospital. J. Pak. Med. Assoc., 2006, 56(9), 409-412.

[8] Radford, DJ. Traumatic false aneurysm in the popliteal artery of a child. Med J Aust 2001, 175(11-12), 658.

[9] Proschek, D.; Proschek, P.; Hochmuth, K.; Balzer, J.O.; Mack, M.G.; Vogl, T.J. False aneurysm of the left femoral artery and thrombosis of the left femoral vein after total hip arthroplasty. Arch. Orthop. Trauma. Surg., 2006, 126(7), 493-497.

[10] D'Angelo, F.; Carrafiello, G.P.; Lagana, D.; Reggiori, A.; Giorgianni, A.; Zatti, G.; Fugazzola, C. Popliteal artery pseudoaneurysm after a revision of total knee arthroplasty: endovascular treatment with a stent graft. Emerg. Radiol., 2007, 13(6), 323-327.

[11] Ersozlu, S.; Ozulku, M.; Yildirim, E.; Tandogan, R. Common peroneal nerve palsy from an untreated popliteal pseudoaneurysm after penetrating injury. J. Vasc. Surg., 2007, 45(2), 408-410.

[12] Sie, R.B.; Dawson, I.; van Baalen, J.M.; Schultze, K.L.J.; van Bockel, J.H. Ruptured popliteal artery aneurysm. An insidious complication. Eur. J. Vasc. Endovasc. Surg., 1997, 13, 432-438.

[13] Harris, A.M.; Barrie, W.W. Deep vein thrombosis: a misdiagnosis of popliteal aneurysms. Br. J. Hosp. Med., 1988, 39(6), 535.

[14] Giustra, P.E.; Root, J.A.; Mason, S.E.; Killoran, P.J. Popliteal vein thrombosis secondary to ruptured popliteal artery aneurysm. Am. J. Roentgenol., 1978, 130, 25-27.

[15] Alwhouhayb, M.; Howard, A. A false aneurysm mistaken for a DVT after hip surgery. Ulus. Travma. Acil Cerrah Derg., 2005, 11(12), 178-179.

[16] Downing, R.; Grimley, R.P.; Ashton, F.; Slaney, G. Problems in diagnosis of popliteal aneurysms. J. R. Soc. Med., 1985, 78(6), 440-444.

[17] Szilagyi, D.E.; Schwartz, R.L.; Reddy, D.J. Popliteal arterial aneurysms. Arch. Surg., 1981, 116, 724-728. 